\title{
Face Recognition Using Nonlinear Feature Parameter and Artificial Neural Network
}

\author{
N. K. Narayanan \& V. Kabeer ${ }^{1}$ \\ School of Information Science and Technology, Kannur University, \\ Mangattuparamba, Kannur, Kerala 670 567, India. \\ E-mail: csirc@rediffmail.com \\ ${ }^{1}$ E-mail: vkabeer@gmail.com
}

Received: 11-06-2009

Accepted: 26-05-2010

\begin{abstract}
The paper reports a study of nonlinear nature of face image. A novel feature extraction method using state space feature parameter for the recognition of face images is studied. The results of simulation experiments performed on the standard AT \& T face database using both Artificial Neural Network and K-Nearest Neighbour recognition algorithms based on Nonlinear Feature Parameter (NLFP) is also presented. Overall recognition accuracy obtained is better for ANN algorithm and is $98.5 \%$.
\end{abstract}

Keywords: Face recognition, Feature extraction, State space parameters, Fractal dimension, Artificial Neural Network, and Pattern Classification.

\section{Introduction}

Machine simulation of human vision has been a subject of intensive research for scientists and engineers for the last three decades. However automatic face recognition is yet to achieve a completely reliable performance. There are several challenges involved in automatic face recognition -large variation in facial appearance, head size, orientation, changes in illumination and poses, occlusion, presence or absence of structural components etc are some of them to list. The interest devoted to this work is not only by the exciting challenges associated, but also the huge benefits that a Face-recognition system, designed in the context of a commercial application, could bring. Moreover, wide availability of powerful and low-cost desktop and embedded computing systems has also contributed to enormous interest in automatic processing of digital images and videos in a number of applications - Entertainment, Smart cards Information security, Low enforcement and Surveillance are some of them. ${ }^{1,2,3,4}$
Face recognition lies at the core of the discipline of pattern recognition where the objective is to recognize an image of face from a set of face images. A complete face recognition system generally consists of three stages. The first stage involves detecting and localizing the face in arbitrary images. 5, 6, 7,8 The second stage requires extraction of pertinent feature from the localized image obtained in the first stage. Finally, the third stage involves classification of facial images based on derived feature vector obtained in the previous stage. In order to design high accuracy recognition system, the choice of feature extraction method is very crucial. Two main approaches to feature extraction have been extensively used in conventional techniques. ${ }^{4,5}$ The first one is based on extracting structural facial features that are local structures of face images, for example, the shapes of the eyes, nose and mouth. The structure based approaches deals with local information rather than global information, and, therefore is not affected by irrelevant information in an image. However, because of the explicit model of facial features, the structure-based approaches are sensitive to unpredictability of face 
appearance and environmental conditions. The second method is statistical-based approach that extracts features from the entire image and, therefore uses global information rather than local information.

There have been a lot of popular attempts towards automated face recognition which kept the research in the area active and vibrant. Some of them are Eigenfaces (PCA based approach), ${ }^{9,10}$ Independent Component Analysis (ICA), ${ }^{11}$ Linear Discriminant Analysis (LDA), ${ }^{12}$ a specific kind of genetic algorithm called Evolutionary Pursuit (EP), ${ }^{13}$ Elastic Bunch Graph Matching (EBGM) where faces are represented as graphs, with nodes positioned at fiducial points, ${ }^{14}$ Kernel Methods which are a generalization of linear methods $^{15}$ like KPCA, KLDA, KICA etc., Trace transform, a generalization of the Radon transform, ${ }^{16}$ Active Appearance Model (AAM) is an integrated statistical model which combines a model of shape variation with a model of the appearance variations in a shape-normalized frame, ${ }^{17}$ Hidden Markov Models (HMM) ${ }^{18}$ and Support Vector Machine (SVM). ${ }^{19}$

There were a few attempts to face recognition using fractal coding methods ${ }^{20,21,22,23}$ where fractal neighbour distance or fractal transformations are employed. The characterizations of speech and image signals as nonlinear chaotic system are also present in literatures. ${ }^{24,25,26}$ Modeling of faces as nonlinear chaotic system using state space pixel distribution is a relatively new signal analysis method. Results of preliminary attempt by the authors in face recognition have already been reported in Ref. 27. The present paper reports the results of a detailed study of application of nonlinear modeling of face images namely state space point distribution or Nonlinear feature Parameter (NLFP) for fruitful application in face recognition. Also, we have made an exhaustive study and provided more evidences of empirical results obtained. In the present work we employed a vectorized representation of pixel gray values to model the face images. The state space maps of the faces are then constructed based on time series relationship between each pixel and the next one. The paper shows that the fractal dimension of the state space map is a fraction by employing box-count method and hence face images are nonlinear. Also, the paper presents a feature representation based on the state space point distribution of pixel intensities. Further a comparative study of the recognition results using Artificial Neural Network (ANN), a nonlinear recognition algorithm and K-Nearest Neighbour (KNN), a classical distance measure algorithm based classifiers is made using the derived feature parameter.

The paper is organized as follows. Section 2 gives the nonlinear analysis and modeling of face images using State Space Map (SSM). Section 2.1 gives the detailed description of the SSM method and 2.2 a preliminary analysis on their fractal dimensions. In section 3 the feature exaction method based on the State Space Point Distribution (SSPD) of the pixel intensities of the face image is explained. Section 4 describes face image classification using artificial neural network and k-nearest neighbour classifiers. Section 5 gives a small account of properties of AT \& T face database and reports the recognition results obtained using ANN and KNN classifiers. Finally, section 6 gives the conclusions and directions for future research.

\section{Nonlinearity Study of Face Images and State Space Map (SSM)}

This section gives a description on nonlinearity, a unique characteristic of dynamic systems, a study carried out on face images. We can show that the face images are characterized by the conditions of a nonlinear chaotic system. To show the nonlinear nature of the face images the fractal dimension is computed from the sate space map of the each face images using the box count method.

\subsection{State space map (SSM) of human face images}

Let $\mathbf{I}_{\mathrm{mn}}$ is an image of size $\mathrm{m} \mathbf{x} \mathrm{n}$, and $\mathrm{X}$ is a set of all pixels in $\mathbf{I}_{\mathrm{mn}}$ such that,

$$
\mathbf{X}=\bigcup_{i=1}^{m} \bigcup_{j=1}^{n}\left\{I_{i, j}\right\}
$$

Then State Space Map can be obtained by plotting a graph with coordinate values $\left(x_{\mathrm{p}}, y_{\mathrm{q}}\right)$, where

$$
y_{q}=\left\{\begin{array}{l}
{ }^{x} p+1, \text { if } 1 \leq p \leq m n-1 \\
{ }^{x} p-m n+1, \text { otherwise }
\end{array}\right.
$$

The state space maps for the PGM formatted image samples of the face images taken from the image database are constructed in the following fashion. Initially, to bring size uniformity among the face samples they are normalized into 100x90(not a 
mandatory step) pixel-sizes using affine transformation and bilinear interpolation algorithm. The information content of the PGM image file is then extracted and the extracted gray level intensity value of each pixel is stored in a separate data file. The state space maps for each face image are then generated for each image file containing 9,000 gray level intensity values. Figure 1 shows the State-Space Map obtained from the grayscale images of the sample face of the first person in AT \& T face database.
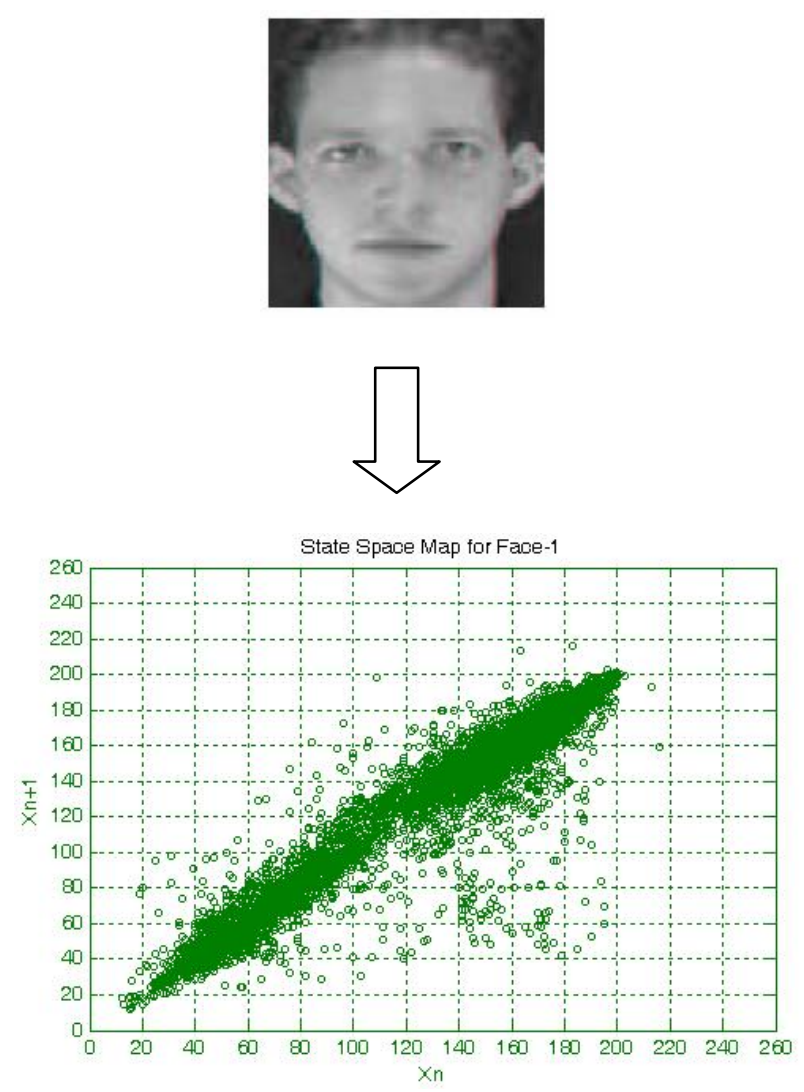

Fig. 1. State space map for face image of a person in AT \& T face database.

\subsection{Fractal dimension of human face images from their State Space Maps}

In this section a preliminary analysis on the nonlinearity nature of faces is presented. For that purpose the fractal dimensions (FD) of the state space map of the each face image is measured. One of the most important methods for computing the fractal dimension of a self-similar fractal is the box counting method. ${ }^{28,29}$ The algorithm used for finding the fractal dimensions of face images based on their state space map using this method is given below.

Step 1: The state-space map of the face image for which the fractal dimension is to be calculated is plotted. (This map may be considered as a binary image structure).

Step 2: N(s), the number of boxes which covers the image content, is calculated by placing a grid on the state space map which divides the map in to boxes of a given size 's'.

Step 3: Repeat the step 2 for different 's'.

Step 4: Plot $\log _{10} \mathrm{~N}$ (s) verses $\log _{10}$ (s) curve for all's'.

Step 5: Find the line of best fit to the curve plotted in the step 4, and find the slope of the line which indicates the fractal dimension of the given structure which is theoretically given by the formula,

$$
D=-\operatorname{Lim}_{s \rightarrow 0} \frac{\log _{10} N(s)}{\log _{10} s}
$$

Table 1. The number of boxes counted for each of the box sizes for SSM of the face image of the first person in AT \& T face.

\begin{tabular}{ccc}
\hline S.No. & $\begin{array}{c}\text { Box sizes } \\
\text { (in pixels) }\end{array}$ & $\begin{array}{c}\text { Number of boxes } \\
\text { containing the image } \\
\text { structure }\end{array}$ \\
\hline 1 & 2 & 1774 \\
2 & 3 & 1094 \\
3 & 6 & 438 \\
4 & 12 & 173 \\
5 & 22 & 65 \\
6 & 40 & 24 \\
7 & 75 & 8 \\
8 & 100 & 3 \\
\hline
\end{tabular}

We have used box sizes, $s=2,3,6,12,22,40,75$ and 100 pixels and plotted the values 's' and $\mathrm{N}(\mathrm{s})$ by taking $\log (s)$ in $\mathbf{x}$-axis and $\log N(s)$ in $\mathbf{y}$-axis. Table 1 shows the number of boxes counted for each of these box sizes for the state space map of the face image of the first person in AT \& T face database. Figure 2 shows the $\log (s)$ vs $\log (N(s))$ plot for the state space map of the face image of the first person in AT \& T face database. 


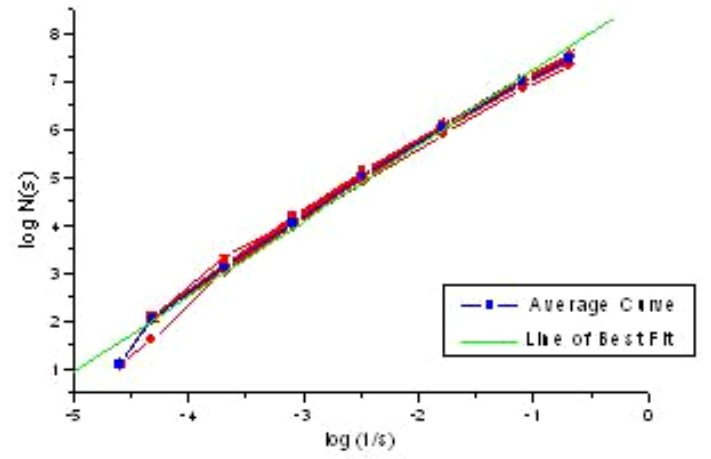

Fig. 2. The $\log _{10}(s)$ vs $\log _{10}(N(s))$ plot for the SSM of face image of the person in AT \& T face database

To fit a straight line for the average curve obtained from different image samples in the above plot, we used the method of least squares procedure by Gauss. ${ }^{30}$ Finally the fractal dimensions of the face images are estimated by finding the slopes of these lines. The fractal dimension of the selected face images estimated from their state space maps using the box-counting method is given in Table 2. From the table it is evident that all fractal dimensions are fractions which show face images are nonlinear in nature.

Table 2. Fractal dimensions of a selected face images in AT $\& \mathrm{~T}$ face database.

\begin{tabular}{ccc}
\hline S.No. & $\begin{array}{c}\text { Person } \\
\text { Class }\end{array}$ & $\begin{array}{c}\text { Fractal } \\
\text { Dimension }\end{array}$ \\
\hline 1 & 1 & 1.576880 \\
2 & 2 & 1.623822 \\
3 & 3 & 1.610308 \\
4 & 4 & 1.623794 \\
5 & 5 & 1.560618 \\
6 & 6 & 1.619526 \\
7 & 7 & 1.639164 \\
8 & 8 & 1.696362 \\
9 & 9 & 1.678693 \\
10 & 10 & 1.653500 \\
\hline
\end{tabular}

\section{Feature Extraction from State Space Point Distribution (SSPD)}

The state space map constructed by extracting and assembling the state space characteristics of the face image pattern surface is then divided into a grid with $16 \times 16$ locations as shown in figure 3. The locations defined by the co-ordinates $(16,0),(0,16)$ is taken as location one and location just right side to it is taken as location two and it is extended towards $\mathrm{X}$ direction with the last box being $(256,0),(240,16)$. Then the next row is scanned from the beginning and locations are numbered accordingly. The same process is repeated for all rows and 256 locations are spotted. As described in Section 2 a digitized grayscale image of the face is viewed as a distribution of the foreground as well as background information. The state space point distribution (SSPD) for each pattern is calculated by estimating the number of points in each of the 256 locations of the state space map.

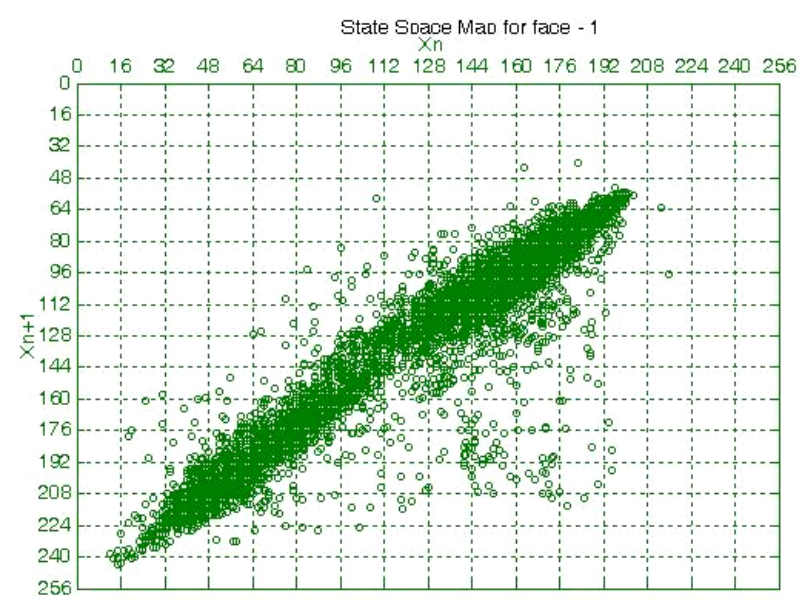

Fig. 3. SSM with $16 \times 16$ locations of a sample face imageof the first person in AT \& T face database.

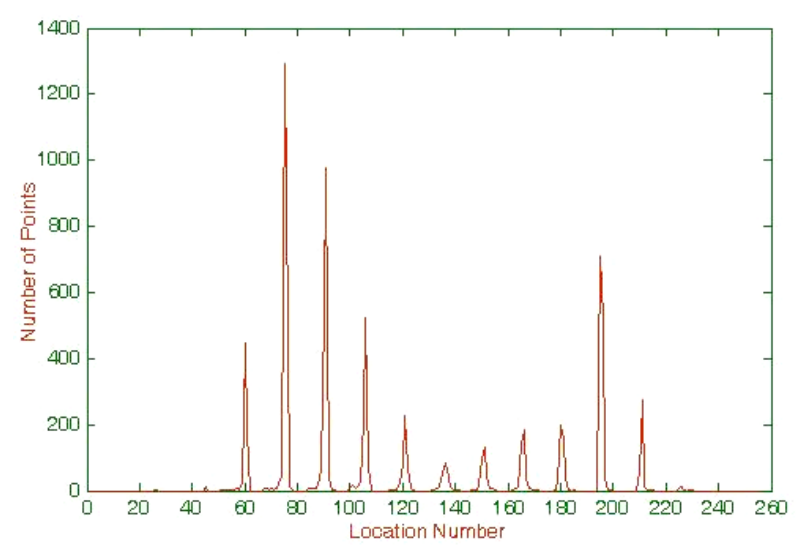

Fig. 4. The $\log _{10}(s)$ vs $\log _{10}(N(s))$ plot for the SSM of face image of the person in AT \& T face database

The state space map and its corresponding state space point distribution graph obtained for different persons show the identity of the face. From this analysis it is found that an efficient feature vector can be formed 
based on the SSPD which in turn can be used for recognition purpose. The feature vector of size 16 is estimated by taking the average distribution of each row in the SSPD graph. Figure 5 shows the feature vector graph plotted for 10 samples of the first person in AT \& $\mathrm{T}$ face database. The graphs obtained from the samples of an individual are found to be similar whereas the graphs for different individuals are found to be highly distinguishable. The recognition based on the SSPD feature is explained in succeeding section.

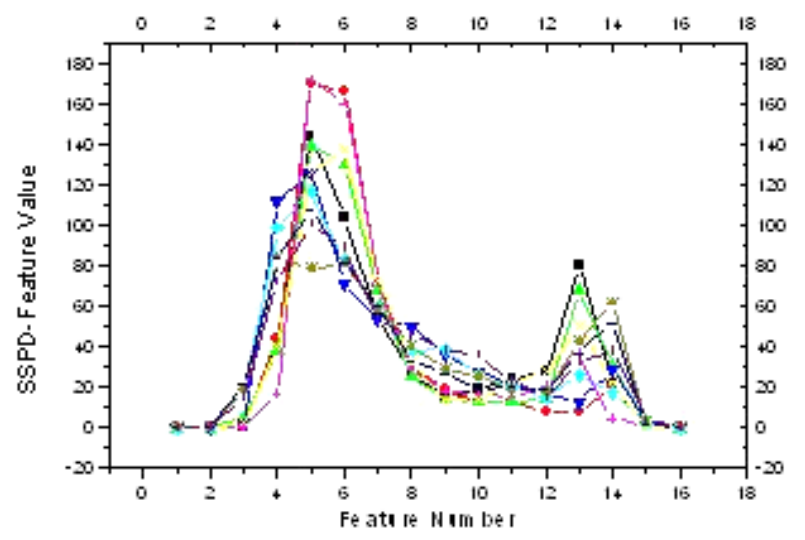

Fig. 5. The feature vector graph plotted for 10 samples of the first person in AT \& T face database.

\section{Classification Using Artificial Neural Network and K-Nearest Neighbour Classifiers}

Pattern classification by distance functions ${ }^{31}$ is one of the earliest concepts in pattern recognition. ${ }^{32,33,34}$ Here the proximity of an unknown pattern to a class serves as a measure for its classification. Pattern classification using neural networks have been in use for more than a few decades now. The classification using neural networks is much efficient than distance measure classifiers. ${ }^{31,41,43}$

\subsection{Artificial Neural Networks (ANN)}

Artificial Neural Networks (ANN) are massively parallel adaptive networks of simple non-linear computing elements called neurons which are intended to abstract and model some functionalities of human nervous system in an attempt to partially capture some of its computational strength. ${ }^{35}$ In other words, a set of processing units when assembled in a closely interconnected network, offers a surprisingly rich structure exhibiting some features of the biological neural network. ${ }^{36}$ Such a structure is called Artificial Neural Network. Artificial neural networks are useful only when the processing units are organized in a suitable manner to accomplish a given pattern recognition task. ${ }^{36}$

Artificial neural networks are normally organized into layers of processing units. The units of a layer are similar in the sense that they all have the same activation dynamics and output function. While developing artificial neural networks for specific applications, the weights are adjusted in a systematic manner using learning laws. The most popular class of multilayer feed-forward networks is multilayer perceptrons in which each computational unit employs either the thresholding function or the sigmoid function. Multilayer perceptrons can form arbitrarily complex decision boundaries and represent any Boolean function. ${ }^{37}$ The development of the back-propagation learning algorithm for determining weights in a multilayer perceptron has made these networks the most popular among researchers and users of neural networks. Let $\mathrm{w}_{\mathrm{ij}}{ }^{(l)}$ denote as the weight on the connection between the $\mathrm{i}^{\text {th }}$ unit in layer $(l-1)$ to $j^{\text {th }}$ unit in layer $l$. Let $\left\{\left(\mathbf{x}^{(1)}, \mathbf{d}^{(1)}\right),\left(\mathbf{x}^{(2)}, \mathbf{d}^{(2)}\right), \ldots,\left(\mathbf{x}^{(\mathbf{p})}, \mathbf{d}^{(\mathrm{p})}\right)\right\}$ be a set of $p$ training patterns (input-output pairs), where $\mathbf{x}^{(\mathrm{i})}$ $\in R^{n}$ is the input vector in the $\mathrm{n}$-dimensional pattern space, and $\mathbf{d}^{(\mathbf{l})} \in[0,1]^{\mathrm{m}}$, an m-dimensional hypercube. For classification purposes, $m$ is the number of classes. The squared error cost function most frequently used in the ANN literature is defined as

$$
E=\frac{1}{2} \sum_{i=1}^{p}\left\|y^{(l)}-d^{(l)}\right\|^{2}
$$

Here, we used a Multi-layer feed forward network having a Gradient descent with momentum, and an adaptive learning rate back propagation learning algorithm. ${ }^{36}$

\subsection{Classification using $k$-NN Classifier}

The k-Nearest-Neighbor ${ }^{38}$ method is a well-known non-parametric classifier, where a posteriori probability is estimated from the frequency of nearest neighbours of the unknown pattern. It considers multiple prototypes while making a decision and uses a piecewise linear discriminant function. 
Let us consider the case of $\mathrm{m}$ classes $\left\{C_{i}\right\}$ where $i=$ 1: $m$ and a set of $N$ patterns $\left\{\mathrm{y}_{i}\right\}$ where $i=1: N$ whose classification is priory known. Let $\mathrm{x}$ denote an arbitrary incoming pattern. The nearest neighbour classification approach classifies $\mathrm{x}$ in the pattern class of its nearest neighbour in the set $\left\{\mathrm{y}_{i}\right\}$ where $i=1$ : N, i.e.

if $\left\|\mathrm{x}-\mathrm{y}_{j}\right\|=\min \left\|\mathrm{x}-\mathrm{y}_{j}\right\| 1 \leq \mathrm{i} \leq \mathrm{N}$ then $\mathrm{x} \varepsilon \mathrm{C}_{j}$.

This scheme (can be termed the $1-\mathrm{NN}$ rule since it employs only the classification of the nearest neighbour to $\mathrm{x}$ ) can be modified by considering the $\mathrm{k}$ nearest neighbours to $\mathrm{x}$ and using a majority-rule type classifier. The following algorithm summarizes the classification process.

Algorithm: Minimum distance k -Nearest Neighbour Classifier.

Input: $\mathrm{n}$ - problem's dimension.

$\mathrm{N}$ - the number of pre-classified patterns

$\mathrm{m}-$ the number of pattern classes.

$\left(x_{i}, j_{i}\right), 1 \leq \mathrm{i} \leq \mathrm{N}-\mathrm{N}$ ordered pairs, where $\mathrm{x}_{\mathrm{i}}$ is the $\mathrm{i}^{\text {th }}$ pre-classified pattern and $\mathrm{j}_{\mathrm{i}}$ its class number $\left(1 \leq \mathrm{j}_{\mathrm{i}} \leq \mathrm{m}\right.$ for all $\left.\mathrm{i}\right)$.

$\mathrm{k}$-the order of $\mathrm{NN}$ classifier (i.e. the $\mathrm{k}$ closest neighbours to the incoming patterns are considered).

$\mathrm{x}$ - an incoming pattern.

Out put: $\mathrm{L}-$ the number of class into which $\mathrm{x}$ is classified.

Step 1: Set $\mathrm{S}=\left\{\left(\mathrm{x}_{\mathrm{i}}, \mathrm{j}_{\mathrm{i}}\right)\right\} \mathrm{i}=1, \ldots, \mathrm{N}$

Step 2: Find $\left(y, j_{0}\right) \in S$ which satisfies $\|\mathrm{y}-\mathrm{x}\|=\min \|\mathrm{z}-\mathrm{x}\|,(\mathrm{z}, \mathrm{j}) \in \mathrm{S}$.

Step 3: If $\mathrm{k}=1$ set $\mathrm{L}=\mathrm{j}_{0}$ and stop; else initialize an $\mathrm{m}$ - dimensional vector IC:

$\operatorname{IC}\left(\mathrm{i}^{\prime}\right)=0, \mathrm{i}^{\prime} \neq \mathrm{j}_{0}$; IC $\left(\mathrm{j}_{0}\right)=1$ and set $\mathrm{S}=\mathrm{S}-\left\{\left(\mathrm{y}, \mathrm{j}_{0}\right)\right\}$

Step 4: For $\mathrm{i}_{0}=1,2 \ldots, \mathrm{k}-1$ do steps $5-6$

Step 5: Find $\left(y, j_{0}\right) \in S$ such that $\|\mathrm{y}-\mathrm{x}\|=\min \|\mathrm{z}-\mathrm{x}\|,(\mathrm{z}, \mathrm{j}) \in \mathrm{S}$.

Step 6: Set $\operatorname{IC}\left(\mathrm{j}_{0}\right)=\operatorname{IC}\left(\mathrm{j}_{0}\right)+1$ and $\mathrm{S}=\mathrm{S}-\left\{\left(\mathrm{y}, \mathrm{j}_{0}\right)\right\}$.

Step 7: Set $\mathrm{L}=\max \{\mathrm{IC}(\mathrm{i})\}, 1 \leq \mathrm{i} \leq \mathrm{m}$ and stop.

The following section gives a detailed description about the experiments we conducted and the classification results obtained.

\section{Experiments and Results}

All the experiments were carried out using the AT \& $\mathrm{T}$ face database, which contains face images of 40 distinct persons. Each person has ten different images, taken at different times. Figure 6 shows five individuals (in five rows) in the AT \& $\mathrm{T}$ face images.

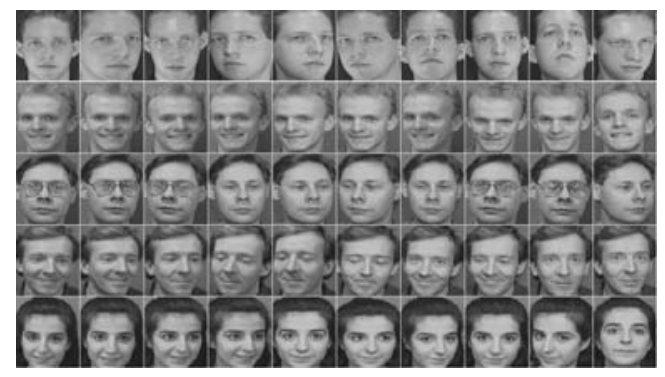

Fig. 6. Sample face images taken from the AT \& $\mathrm{T}$ face Database.

There are variations in facial expressions such as open/closed eyes, smiling/non-smiling, and facial details such as with glasses/without glasses. All the images were taken against a dark homogeneous background with the subjects in an up-right, frontal position, with tolerance for some side movements. There are also some variations in scale.
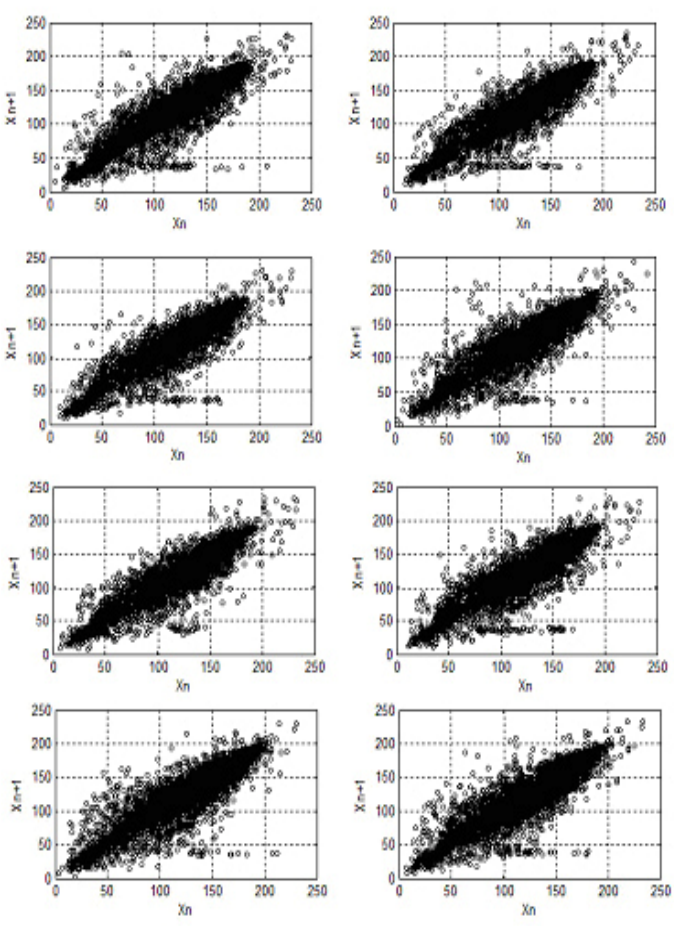

Fig. 7: State space map of eight instances of face image of the given person in AT \& T face database. 

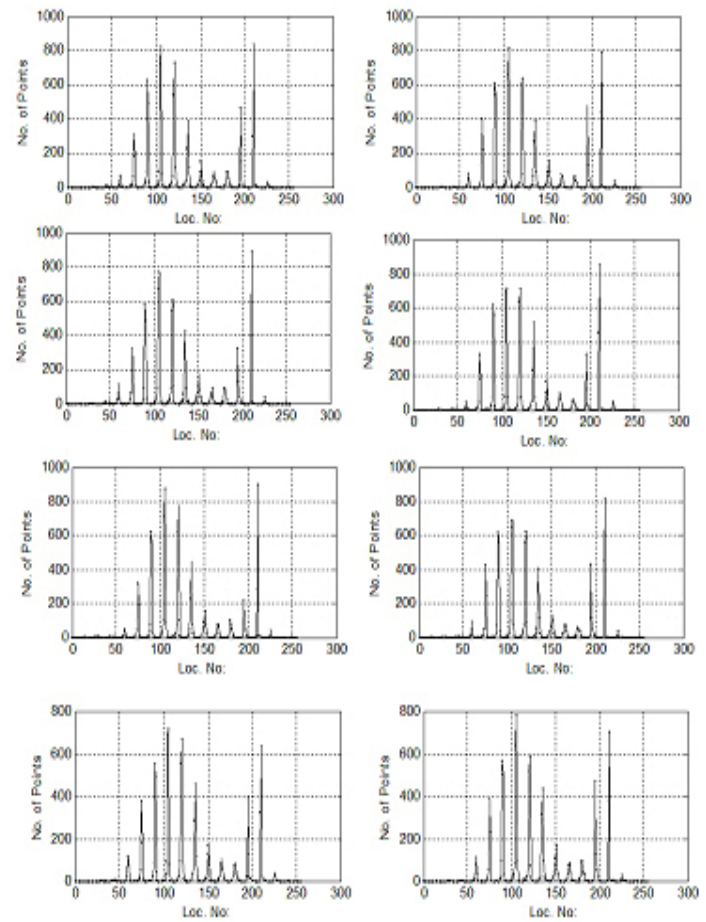

Fig. 8: State space point distribution graphs of eight instances of face image of the given person in AT \& $\mathrm{T}$ face database.

As described in section 2 and 3 we have modelled and characterized face images using state space map(SSM) and state space point distribution (SSPD) graph. Nonlinear Feature vectors are then derived based on SSPD. To analyze the elegance of the method we show SSM and SSPD of eight instances of a given person in figures 7 and 8 respectively. Observation of these graphs reveals that shift, shape and spread/concentrations of the point distribution are very close and similar and hence they represent a single person.

The figures 9 and 10 show the SSM and SSPD respectively of eight different persons in the AT \& T face database. Considerable amount of change in shift, shape and spread/concentration of SSM and SSPD shows the difference in class or object group under consideration. Hence the representation can effectively be used for deriving features for the classification purpose. In the recognition stage we used ANN and KNN classifiers. Figure 11 gives recognition accuracies for forty persons in the standard AT \& $\mathrm{T}$ face image database using both the classifiers.
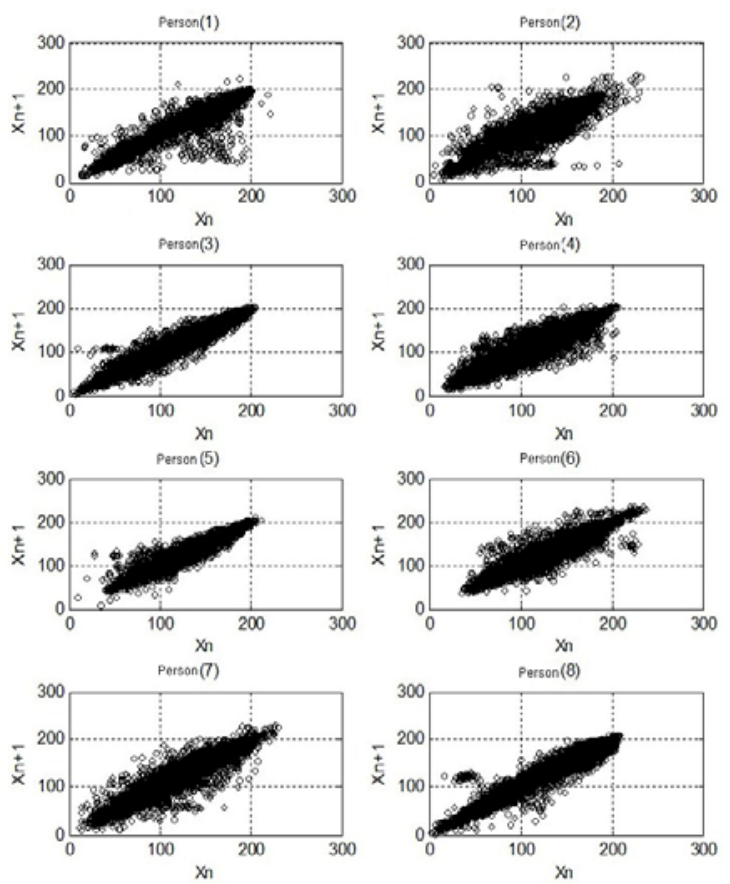

Fig. 9: State space map of first 8 persons' face image in AT \& T face database.
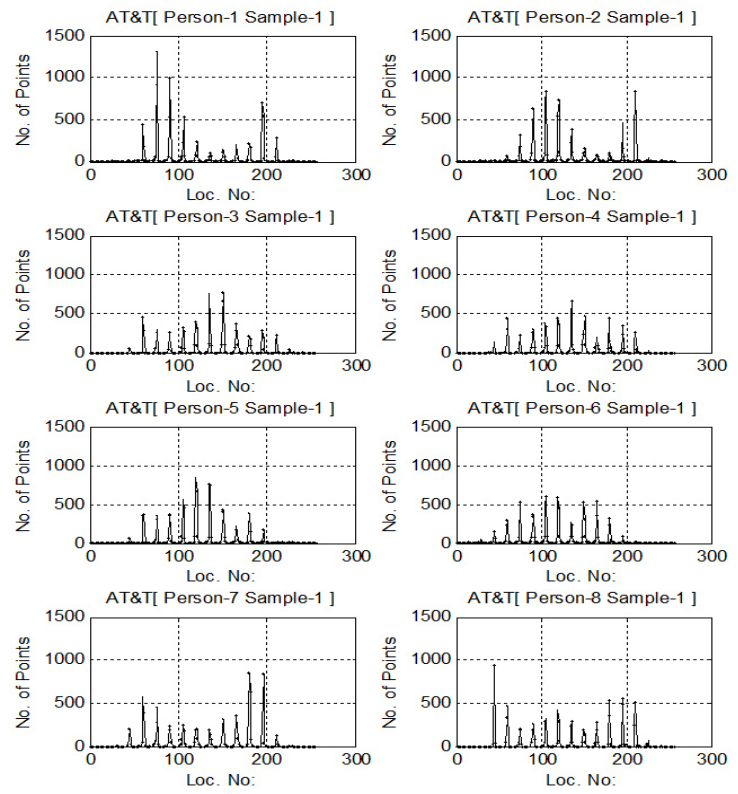

Fig. 10: State space point distribution graphs of first 8 persons' face image in AT \& T face database 


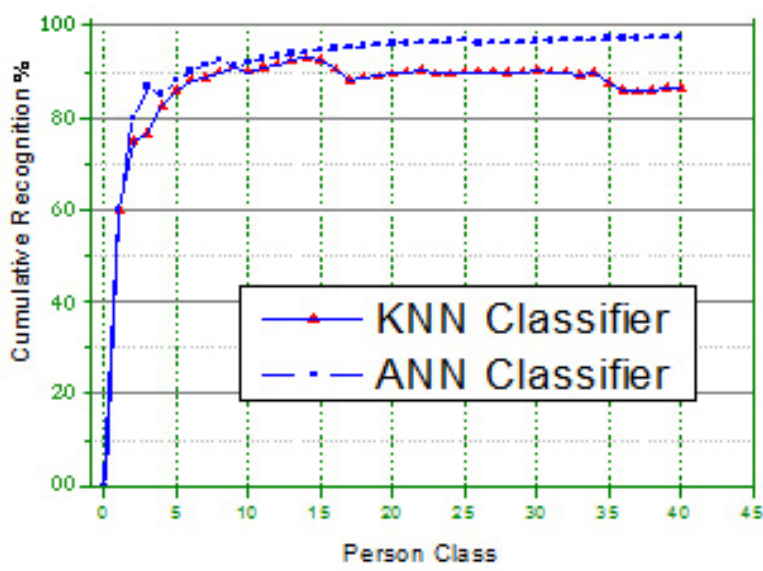

Fig. 11: Face Recognition Performance State Space Feature parameter on AT \& $\mathrm{T}$ face database using ANN and KNN Classifiers.

Overall recognition obtained using nonlinear feature parameter (NLFP) with ANN classifier is $98.5 \%$ and that of $\mathrm{KNN}$ is $85 \%$ this result is a better one compared to other popular methods reported in literatures. Table 3 summarizes this.

Table 3. Comparison of NLFP method with other popular methods reported

\begin{tabular}{clc}
\hline S.No. & Method & Recognition rate \\
\hline 1 & NLFP & 98.50 \\
2 & PCA & 89.50 \\
3 & Chen-LDA & 77.53 \\
4 & D-LDA & 89.50 \\
5 & ICA & 84.50 \\
\hline
\end{tabular}

\section{Conclusion}

This paper presented a novel approach to model face images based on the state space map and state space point distribution of the gray scale images for the computer recognition of human faces. The state space map and state space point distribution graph for each face image is drawn and the fractal dimension of the face image is also estimated from their state space map using box-counting method. Finally, a feature vector named Nonlinear Feature Parameter of size 16 based on the SSPD is formed for face images of each person and recognition accuracies are computed and compared using ANN and KNN classifiers. Present study shows that all the fractal dimensions computed are fractions which prove the chaotic nature of the face images. There is significant dimensionality reduction as we used a feature vector of 16-element size to represent a face image which reduces the computational complexity. Further the recognition accuracy is better (98.5\%) compared to other methods reported in the literatures, More effective implementation using higher dimensional state space and multiple classifier system is one of our future research directions.

\section{References}

1. W. Zhao, R. Chellappa, P.J Philips and A. Rosenfeld, Face Recognition: A Literature Survey, ACM Computing Surveys, 35(4) (2003), 399-458.

2. Ming-Hsuan Yang, David J Kriegman and Narendra Ahuja, Detecting faces in Images: A Survey, IEEE Transactions on Pattern Analysis and Machine Intelligence, 24(1) (2002), 34-58.

3. M.A. Grudin, On internal representation of face recognition systems, Pattern Recognition, 33(7) (2000), 1161-1177.

4. J. Daugman, Face detection: A survey, Computer Vision and Image Understanding, 83(3) (2001), 236-274.

5. Stan Z. Li, Anil K. Jain, Handbook of Face Recognition (Springer, 2004).

6. J. Haddadnia and M. Ahmadi, N-feature neural network human face recognition, Image and Vision Computing, 22 (2004), 1071-1082.

7. J. Haddadnia , M. Ahmadi, K Faez, An Efficient Method for Recognition of Human face using high order Pseudo Zernike Moment Invariant, The 5th IEEE International Conference on Automatic Face and Gesture Recognition, (Washington DC,USA, May 20-21, 2000), pp.315-332.

8. J. Wang, T. Tan, 'A new face detection method based on shape information', Pattern Recognition Letter, 21(2000), 463-471.

9. M. Turk, A. Pentland, Eigenfaces for Recognition, Journal of Cognitive Neurosicence, 3(1) (1991), 71-86.

10. H. Moon, P.J. Phillips, Computational and Performance aspects of PCA-based Face Recognition Algorithms, Perception, 30(2001), 303-321.

11. M.S. Bartlett, J.R. Movellan, T.J. Sejnowski, Face Recognition by Independent Component Analysis, IEEE Trans. on Neural Networks, 13(6) (2002), 1450- 1464.

12. K. Etemad, R. Chellappa, Discriminant Analysis for Recognition of Human Face Images, Journal of the Optical Society of America, 14(8) (1997), 1724-1733.

13. C. Liu, H. Wechsler, Evolutionary Pursuit and Its Application to Face Recognition, IEEE Trans. on Pattern Analysis and Machine Intelligence, 22(6) (2000), 570582.

14. L. Wiskott, J.-M. Fellous, N. Krueuger, C. von der Malsburg, Face Recognition by Elastic Bunch Graph Matching, (Chapter 11 in Intelligent Biometric 
Techniques in Fingerprint and Face Recognition, eds. L.C. Jain et al., CRC Press, 1999).

15. M.-H. Yang, Kernel Eigenfaces vs. Kernel Fisherfaces: Face Recognition Using Kernel Methods, Proc. of the Fifth IEEE International Conference on Automatic Face and Gesture Recognition (Washington D.C., USA, 20-21 May 2002) pp.215-220.

16. A.Kadyrov, M. Petrou., The Trace Transform and Its Applications, IEEE Transactions on Pattern Analysis and Machine Intelligence, 23(8) (2001), 811-828.

17. T.F. Cootes, K. Walker, C.J. Taylor, View-Based Active Appearance Models, Proc. of the IEEE International Conference on Automatic Face and Gesture Recognition (Grenoble, France 26-30 March 2000) ,pp. 227-232.

18. A.V. Nefian, M.H. Hayes, Maximum likelihood training of the embedded HMM for face detection and recognition, Proc. of the IEEE International Conference on Image Processing, ICIP 2000, (Vancouver, BC, Canada 10 - 13 September 2000) Vol. 1, pp. 33-36.

19. G. Guo, S.Z. Li, K. Chan, Face Recognition by Support Vector Machines, Proc. of the IEEE International Conference on Automatic Face and Gesture Recognition, (Grenoble, France 26-30 March 2000), pp.196-201.

20. T. Tan and H. Yan. Face recognition using the weighted fractal neighbor distance. IEEE Trans. Systems, Man, and Cybernetics, 35(4) (2005), 576 - 582.

21. T. Tan and H. Yan. Object recognition based on fractal neighbor distance. Signal Processing, 81(2001), 21052129.

22. T. Tan and H. Yan. The fractal neighbor distance measure. Pattern Recognition, 35(2002), 1371-1387,

23. Andrea F. Abate, Riccardo Distasi, Michele Nappi and Daniel Riccio, face authentication using speed fractal technique, Image and Vision Computing, 24(9) (2006), 977-986,

24. V.L Lajish, V Kabeer and N.K. Narayanan, Handwritten Character Recognition Using State-Space Parameters and k-NN Classifier, Proc. of IEEE Int. Conference on Image and Signal Processing, ( Hubli, India, Dec 7-8, 2006).

25. P.Prajith and N.K. Narayanan, Nonlinear phase space features for pitch detection, Proc. of National Symposium on Acoustics (NSA), NPL, (New Delhi, India, November 2006).

26. P.Prajith, N.S.Sreekanth \& N.K. Narayanan, Phase Space parameters for Neural Networks Based Vowel Recognition, Proceedings of the 11th International Conference on Neural Information Processing - ICONIP(New Delhi, India 2004), pp.1204-1209.

27. V Kabeer \& N.K Narayanan, Face recognition using State Space Parameters and Artificial Neural Network Classifier, International Conference on Computational Intelligence and Multimedia, ICCIMA'07,(Sivakasi, India Dec - 2007), pp.250-254

28. Edwart Ott, Chaos in Dynamical Systems, (Cambridge University Press, 1996).

29. Greogory L, Baker \& Jerry Gollub, Chaotic Dynamics an Introduction, (Cambridge University Press, 1996).
30. Erwin Kreyszig, Advanced Engineering Mathematics, (John Wiley and Sam Inc, 1993).

31. J.T. Tou \& R.C Gonzalez, Pattern Recognition principle, (Addison Wesley, London, 1974).

32. Sankar K. Pal and Sushmitha Mitra, Neuro-fuzzy Pattern Recognition - Methods in Soft Computing, (John Wiley \& Sons, Inc., 1999).

33. M. Friedman, A. Kandel, Introduction to Pattern Recognition - Statistical, Structural, Neural and Fuzzy Logic Approach, (WAT \& Td Scientific, 1999).

34. Simon Haykin, Neural Networks a comprehensive foundation, (Pearson Education, 2001).

35. Kohonen T, An Introduction to neural computing, Neural Networks, 1(1988), pp.3-16.

36. B Yegnanarayana, Artificial neural networks, (PHI, New Delhi, 2005).

37. M. Minsky and S. Papert, Perceptrons: An introduction to Computational Geometry,(MIT Press, Cambridge, 1969).

38. Duda, \& Hart, Pattern Classification and Scene Analysis, (Wiley-interscience, 1973).

39. Abdelhamid Bouchachia,Radial Basis Function Nets for Time Series Prediction, IJCIS, Vol: 2 -issue2, pages 147157,June 2009

40. J.A. Antonino-Daviu, M. Riera-Guasp, M. PinedaSanchez, J. Pons-Llinares, R. Puche-Panadero, J. PerezCruz, Feature Extraction for the Prognosis of Electromechanical Faults in Electrical Machines through the DWT, IJCIS , Vol.2 - issue: 2 pp:158 - 167, June 2009.

41. Enrico Zio, Neural Networks Simulation of the Transport of Contaminants in Groundwater, IJCIS, Vol:2 - issue: 3 pp: 267 - 276, October 2009

42. Xian Shen, X.Z. Gao, Roufang Bie, Artificial Immune Networks: Models and Applications, IJCIS, Vol.1 issue: 2 pp: 168 - 176, 2008

43. Gowrishankar, P.S. Satyanarayana, Neural Network Based Traffic Prediction for Wireless Data Networks , IJCIS, Vol:1 - issue:4, pp: 379 - 389, 2008 